\title{
Relationship between Leukocytosis and Ischemic Complications following Aneurysmal Subarachnoid Hemorrhage
}

\author{
Aldo Spallone, M.D., Michele Acqui, M.D., Francesco S. Pastore, M.D., and \\ Beniamino Guidetti, M.D. \\ Division of Neurosurgery, Department of Neurological Sciences, University of Rome School of Medicine, Rome, Italy
}

Spallone A, Acqui M, Pastore FS, Guidetti B. Relationship between leukocytosis and ischemic complications following aneurysmal subarachnoid hemorrhage. Surg Neurol 1987;27:253-8.

\begin{abstract}
The prognostic significance of admission leukocytosis with respect to ischemic complications of subarachnoid hemorrhage was retrospectively investigated in a series of patients with recently ruptured intracranial aneurysms. The present study concerned 47 consecutive cases admitted within 72 hours following the last hemorrhage, in the years 1982-1984. There was no difference in the admission WBC counts between patients who subsequently deteriorated due to ischemic complications and those who did not. However, the cell count rose significantly at the time of the clinical manifestations of ischemia, possibly as a result of structural damage of brain tissue and/or increased sympathetic and adrenocortical activity. The possible contribution of leukocytes to the pathogenesis of ischemic damage following subarachnoid hemorrhageperhaps through the release of leukotrienes-will require further investigation.
\end{abstract}

KEY WORDS: Intracranial aneurysms; Subarachnoid hemorrhage; Cerebral vasospasm; Ischemic complications; Leukocytes; Leukotrienes

The prognostic significance of leukocytosis in the clinical course of aneurysmal subarachnoid hemorrhage ( $\mathrm{SAH}$ ) has received little attention so far. In fact, mild elevation of the white blood cell (WBC) count is a common finding following SAH. A recent study reported a good correlation between the admission WBC counts and the clinical outcome in a series of patients admitted within 48 hours of SAH. In particular, leukocytosis exceeding 20,000 predicted, rather precisely, a poor outcome [12].

In this study, as well as in an earlier study performed by other investigators [11], leukocytosis was observed to rise subsequently in patients deteriorating after their

Address reprint requests to: Dr. Aldo Spallone, Clinical Neurochirurgica Università, Viale Dell'Università, 30, 00185 Roma, Italy. admission. In neither study was the relationship between leukocytosis at the time of admission and subsequent ischemic complications (ICs) following aneurysmal $\mathrm{SAH}$ specifically investigated.

Recent evidence has indicated that neutrophils may play a role in myocardial $[5,7,8,15,16]$ as well as in cerebral [13] ischemic damage, possibly by releasing vasoactive substances such as leukotrienes [10]. Leukotrienes increase vessel permeability [2] and might be implicated in the pathogenesis of postischemic cerebral edema [22]. Therefore, an investigation of the relationship between leukocytosis and ICs of aneurysmal SAH appears to be justified. This is the subject of the present study.

\section{Material and Methods}

The present study concerns 47 consecutive patients with ruptured intracranial aneurysms admitted to the Division of Neurosurgery in the years 1982-1984. For the purpose of this study, we considered only patients admitted within 72 hours following SAH.

The clinical management of these cases followed the guidelines expressed in earlier reports $[3,14,20]$. Briefly, patients were treated with bed rest, steroids, and mild sedation when necessary. Antifibrinolytics (low-dose AMCA + aprotinine) were used preoperatively in patients who were not candidates for early surgery. Antihypertensives were only used occasionally. Ischemic complications were diagnosed on clinical grounds and treated with blood volume expansion and hemodilution. Angiography was performed only for diagnostic purposes and was not repeated in any patient.

For the purpose of this study, the following data were reviewed (Table 1): sex, age, clinical grade at admission, blood pressure at admission, number of SAHs, location of the aneurysm, and WBC count at admission; and during the subsequent clinical course, angiography, timing of surgery (timing and results), occurrence of ICs, and occurrence of infectious complications.

Patients who exhibited a progressive neurological de- 
Table 1. Data Reviewed for the Present Study

\begin{tabular}{ll}
\hline Sex, Age & Angiography \\
Clinical grade at admission & Timing \\
Blood pressure at admission & Results \\
Number of hemorrhages & Surgical timing \\
Location of aneurysm & Ischemic complications \\
White blood cell counts & Infective complications \\
\hline
\end{tabular}

terioration were diagnosed as suffering from ICs after having ruled out other complications such as rebleeding, hydrocephalus, and electrolytic derangement. Seventeen cases $(36 \%)$ fulfilled these criteria and were considered to have had ICs. These and the remaining 30 cases $(64 \%)$ who did not suffer from ICs were considered as separate groups for the purpose of the present study.

A sequential profile of the WBC counts was traced in the group with ICs, and the cell count value observed at the beginning of the clinical deterioration was evaluated. In the other group, we considered for comparison the WBC count values noticed on day 5 following $\mathrm{SAH}$. This represented the mean interval at which the initial manifestations of ischemia were observed in the other patient group.

Vasospasm was evaluated on angiograms according to the following scale: 0 , absent or mild (minimal vessel changes); 2, moderate [localized spasm of one or two major cerebral arteries]; 4 , severe (diffuse spasm of two or more cerebral arteries).

Diagnosis of infective complications required clinical

Table 2. Sex, Age, and Timing of Surgery

\begin{tabular}{lcc}
\hline & Cases with ICs & Cases without ICs \\
\hline Sex & & \\
Male & 10 & 1.4 \\
Female & 7 & 16 \\
Age (years) & $47.1( \pm 10.1)$ & $45.5( \pm 12.3)$ \\
Timing of surgery (days) & $15.9( \pm 13.6)$ & $15.1( \pm 8.4)$ \\
\hline
\end{tabular}

Abbreviation: IC, ischemic complication.

Table 3. Clinical Grade at Admission

\begin{tabular}{lccccc}
\hline & \multicolumn{2}{c}{ Cases with ICs } & & \multicolumn{2}{c}{ Cases without ICs } \\
\cline { 2 - 3 } Clinical grade & $\mathbf{n}$ & $\%$ & & $\mathbf{n}$ & $\%$ \\
\hline I & 7 & 41.2 & & 10 & 33.3 \\
II & 6 & 35.3 & & 12 & 40 \\
III & 3 & 17.6 & & 6 & 20 \\
IV & 1 & 5.9 & 2 & 6.6 \\
Total & 17 & 100 & & 30 & 100 \\
\hline
\end{tabular}

${ }^{\circ}$ Hunt and Hess Scale.

Abbreviation: IC, ischemic complication.
Table 4. White Blood Cell Counts at Admission versus Clinical Grade

\begin{tabular}{lcr}
\hline & \multicolumn{2}{c}{ Mean WBC counts } \\
\cline { 2 - 3 } Clinical grade & \multicolumn{1}{c}{ Cases with ICs } & \multicolumn{1}{c}{ Cases without ICs } \\
\hline I & $9,427 \pm 2,177$ & $8,650 \pm 1,090$ \\
II & $9,500 \pm 1,750$ & $9,917 \pm 2,353$ \\
III & $9,500 \pm 1,871$ & $10,500 \pm 2,550$ \\
IV & 13,000 & $10,750 \pm 250$ \\
\hline
\end{tabular}

Abbreviations: IC, ischemic complication; WBC, white blood cell.

Table 5. Location of Aneurysms

\begin{tabular}{lcc}
\hline & Cases with ICs & Cases without ICs \\
\hline ACoA-ACA & $6(35 \%)$ & $12(40 \%)$ \\
MCA & $5(29 \%)$ & $6(20 \%)$ \\
ICA & $4(24 \%)$ & $5(17 \%)$ \\
VB & $2(12 \%)$ & $2(7 \%)$ \\
Multiples & 0 & $5(17 \%)$ \\
Total & $17(100 \%)$ & $30(100 \%)$ \\
\hline
\end{tabular}

Abbreviations: AcoA, anterior communicating artery; $\mathrm{ACA}$, anterior cerebral artery; MCA, middle cerebral artery; ICA, internal carotid artery; VB, vertebrobasilar system; IC, ischemic complication.

Table 6. Blood Pressure at Admission and Number of Subarachnoid Hemorrbages

\begin{tabular}{lcc}
\hline & Cases with ICs & Cases without ICs \\
\hline Blood pressure & & \\
Systolic & $152 \pm 21^{\prime}$ & $123 \pm 11^{\circ}$ \\
Diastolic & $89 \pm 10$ & $79 \pm 6$ \\
Number of SAHs & $11(65 \%)$ & $23(77 \%)$ \\
Single & $6(35 \%)$ & $7(23 \%)$ \\
Multiple & $17(100 \%)$ & $30(100 \%)$ \\
Total & & \\
\hline &
\end{tabular}

Table 7. Angiograpbic Vasospasm

\begin{tabular}{|c|c|c|}
\hline & Cases with ICs & Cases without ICs \\
\hline \multicolumn{3}{|c|}{ Interial from $S A H$ to angiographic examination } \\
\hline $0-3$ days & $9^{b}(53 \%)$ & $7^{6}(23 \%)$ \\
\hline $4-13$ days & $8(47 \%)$ & $20(67 \%)$ \\
\hline$>14$ days & 0 & $3(10 \%)$ \\
\hline Total & $17(100 \%)$ & $30(100 \%)$ \\
\hline \multicolumn{3}{|l|}{ Angiographic findings } \\
\hline $\begin{array}{l}\text { Absent or mild } \\
\text { vasospasm }\end{array}$ & 12 & 25 \\
\hline Moderate vasospasm & 2 & 3 \\
\hline Severe vasospasm & 3 & 2 \\
\hline Total vasospasm score ${ }^{a}$ & $16^{6}$ & $14^{b}$ \\
\hline
\end{tabular}

${ }^{a}$ See text for explanation.

${ }_{0} \mathrm{p}<0.05$.

Ábbreviations: IC, ischemic complication; SAH, subarachnoid hemorrhage. 

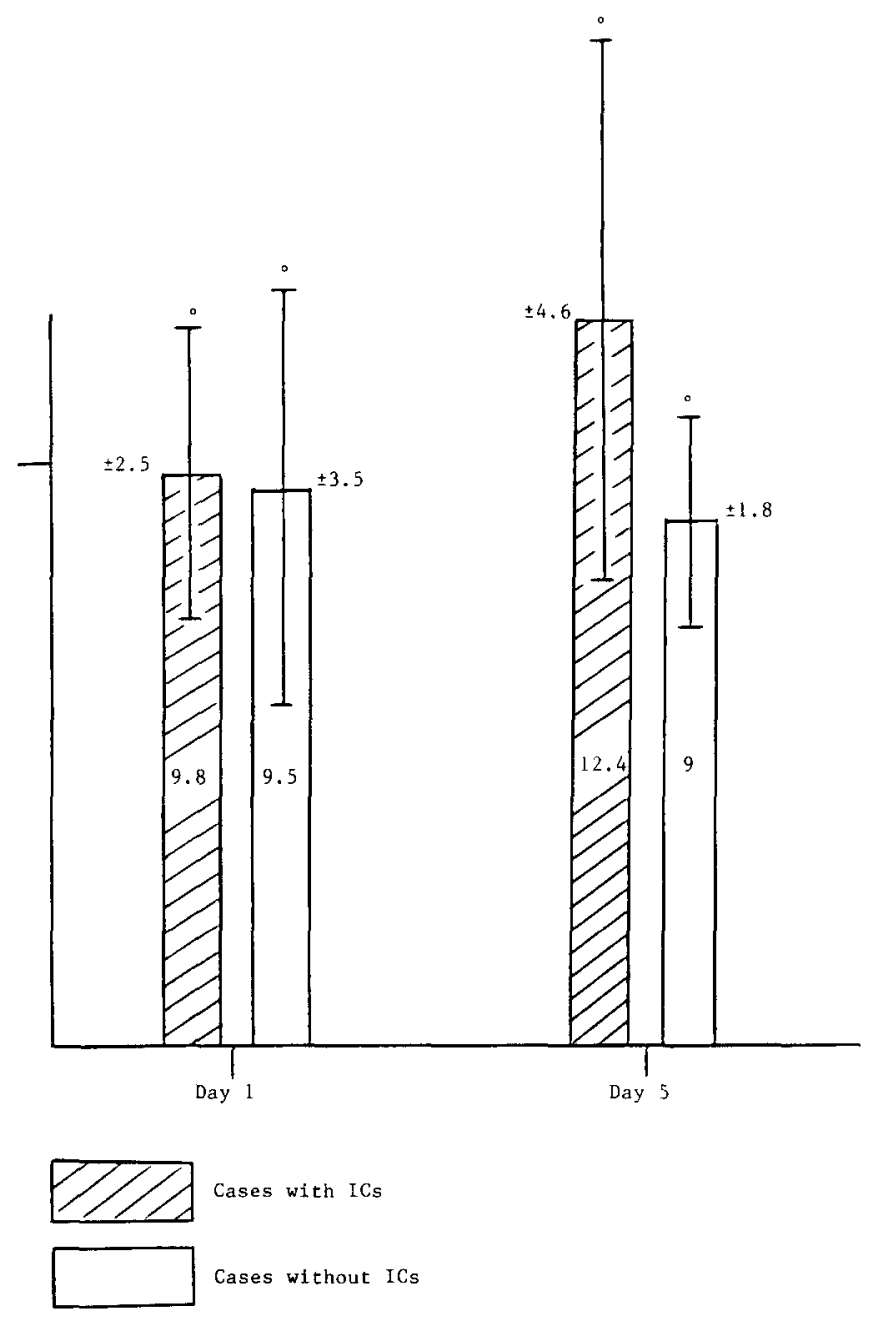

$\circ \mathrm{p}<0.001$

Figure 1. White blood cell (WBC) counts of patients with and withou ischemic complications (ICs).

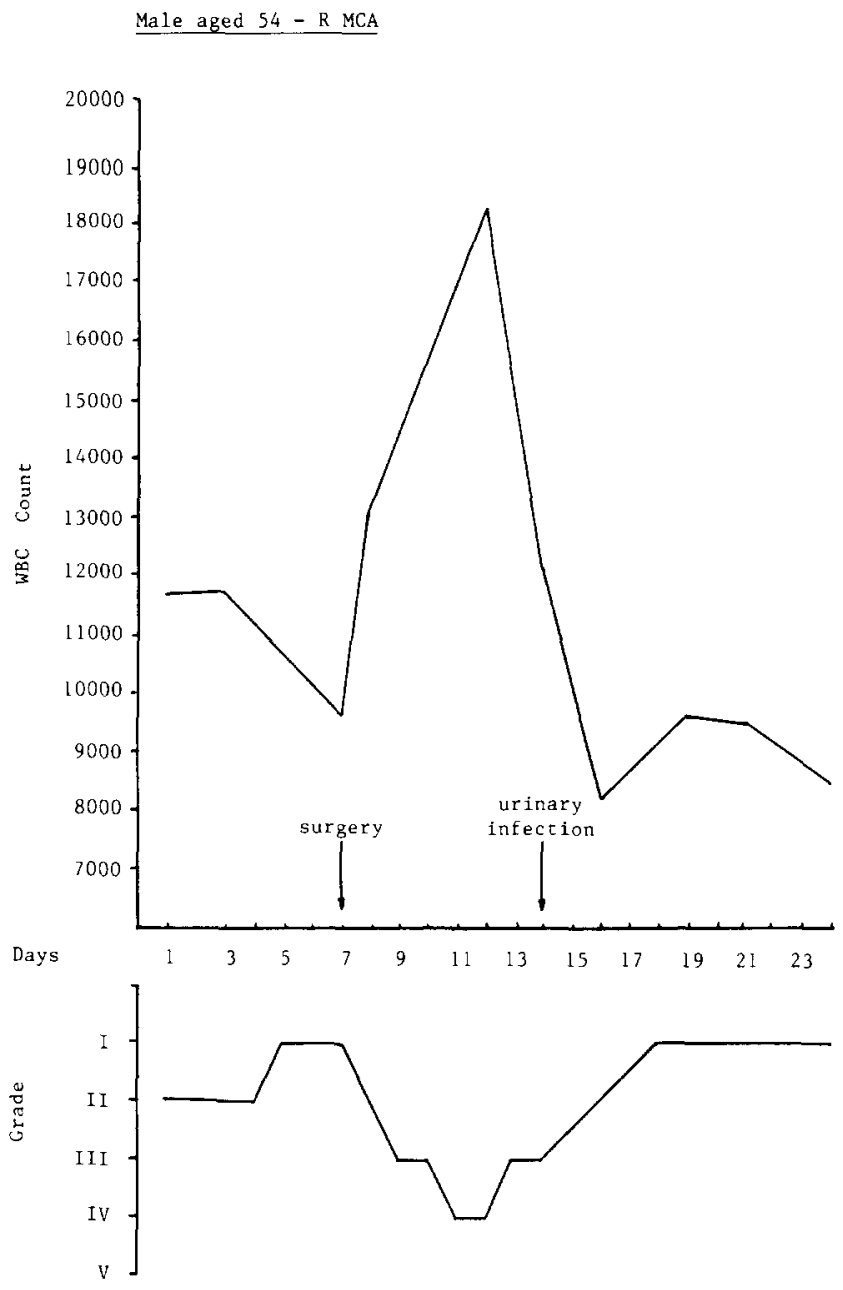

Figure 3. White blood cell (WBC) count and clinical grade of a man aged 54 years with ischemic complications.

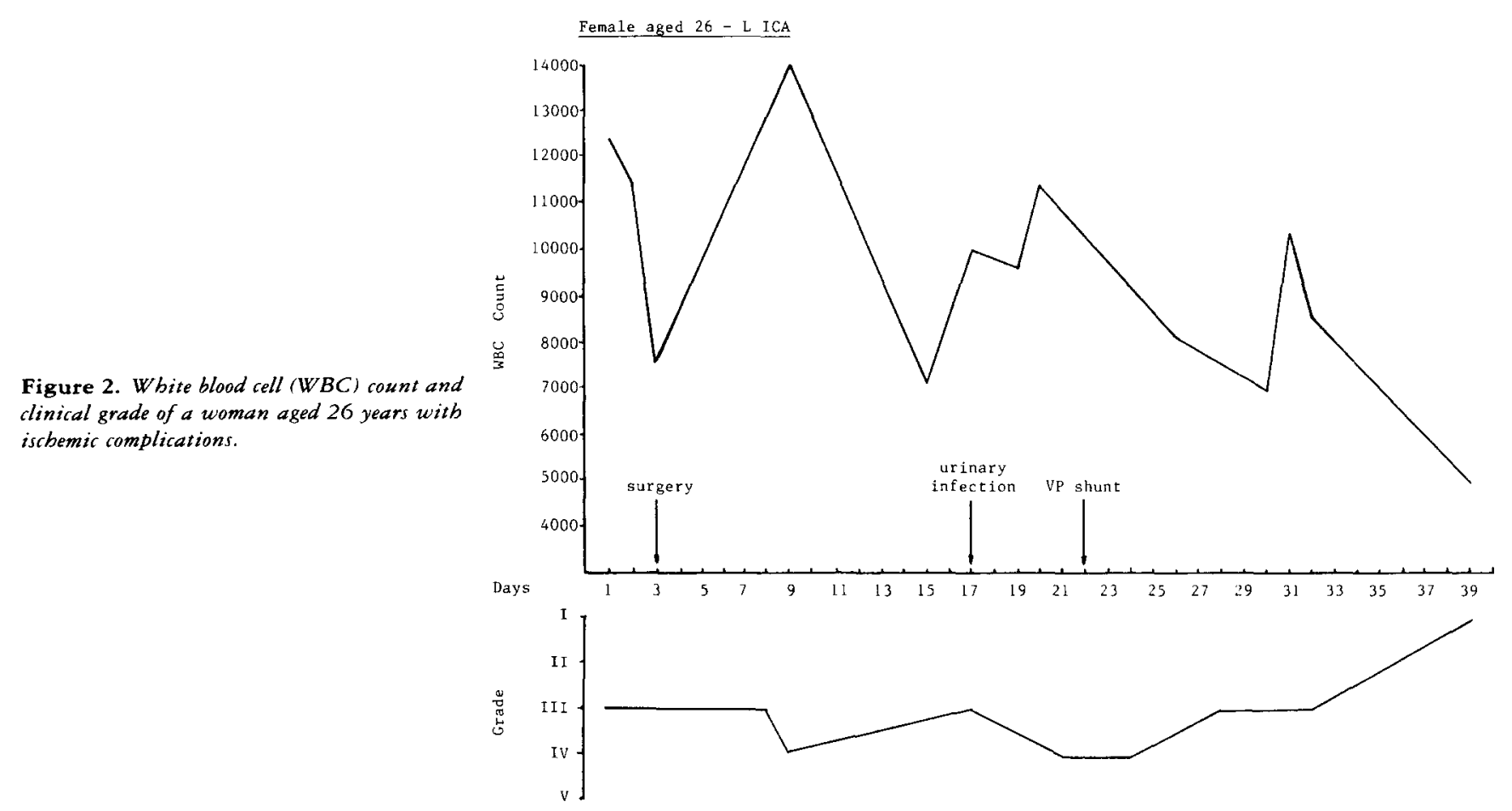




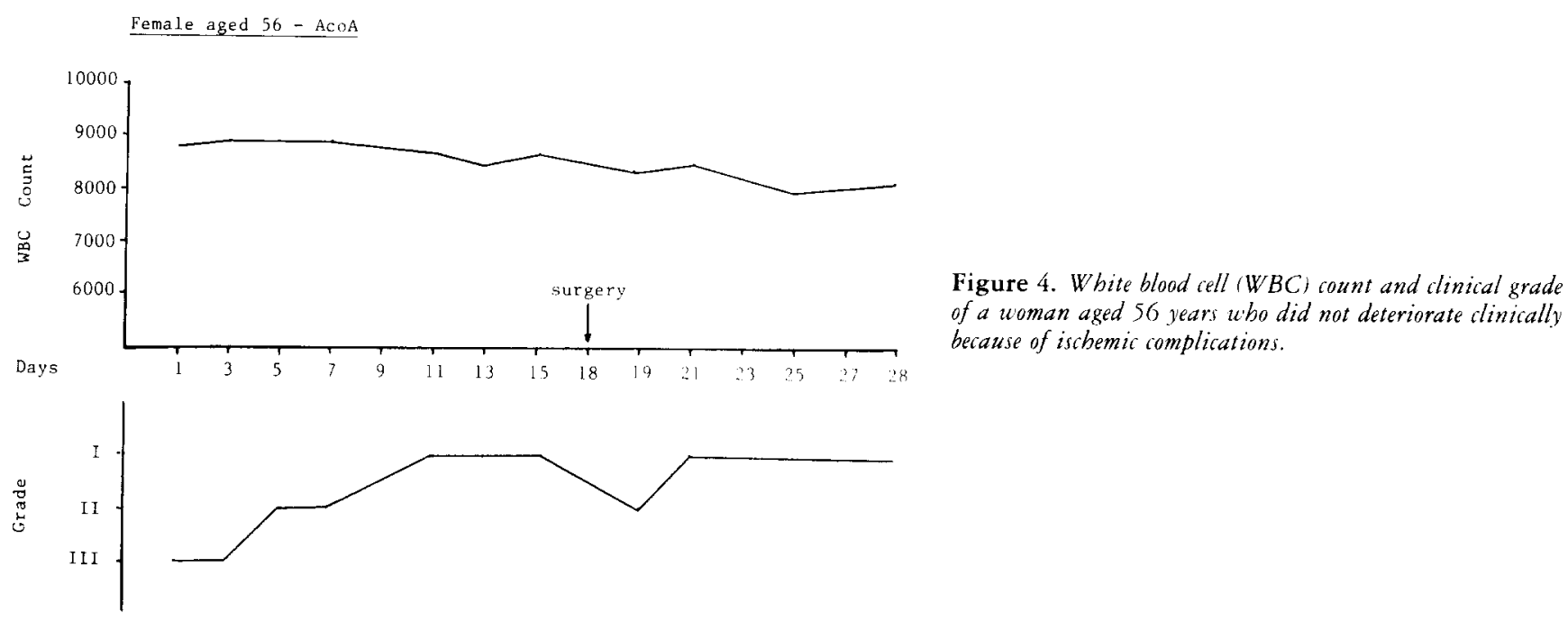

evidence as well as laboratory and bacteriological confirmation. For the present study we did not consider pulmonary infections, as they were a relatively common sequelae of prolonged bed rest in seriously ill patients, and were seldom observed in the first 2 weeks after SAH.

\section{Results}

Sex, age, and timing of surgery were similar in patients with and without ICs (Table 2). Clinical grade at admission was also similar in the two groups of patients (Table 3). The WBC counts on admission were higher in patients graded worse (Table 4).

Concerning the location of the lesions, aneurysms of the internal carotid artery and middle cerebral artery were slightly overrepresented in the group of patients who had ICs, but the difference was not significant (Table 5).

Blood pressure levels were higher in the patients who suffered from ICs and who also had more episodes of SAH (Table 6). The majority of these patients underwent angiographic examination within 3 days of the hemorrhage, when angiographic vasospasm is unlikely. Nevertheless, they had a significantly higher $(\mathrm{p}<0.05)$ score of angiographic vasospasm (Table 7).

There was no difference in the admission WBC count values between patients with and without ICs. However, a statistically significant rise in the cell count was observed at the beginning of the clinical deterioration (mean interval, 5 days after $\mathrm{SAH}$ ) due to ischemia (Figure 1). Serial WBC counts appeared to be roughly correlated with the clinical course in patients suffering from ICs (Figures 2 and 3). An increase in the subsequent WBC count was not observed in patients who did not deteriorate clinically due to ICs (Figure 4).
There were 15 patients with infectious complications; $8(47 \%)$ were in the group with ICs and $7(23 \%)$ were in the group without ICs $(0.3>\mathrm{p}>0.2)$.

All but two patients suffered from urinary infections (there was a case of wound sepsis and a case of cholecystitis). The interval at which the initial manifestations of such a complication were observed was similar in the two groups of patients $(12 \pm 4.5$ days in the group with ICs vs. $11 \pm 5.8$ days in the group without ICs.

\section{Discussion}

The pathogenesis of ICs, presumably due to cerebral vasospasm following aneurysmal $\mathrm{SAH}$, is still far from clear. Recent advances in the study of pharmacological properties of arachidonic acid derivatives have provided some clues for a better understanding of the pathophysiology of cerebral arterial constriction following SAH. It has been suggested that an imbalance in the prostacyclin/thromboxane $A_{2}$ ratio $[6,14,19,21]$, possibly triggered by a free radical reaction initiated by the presence of blood in the subarachnoid spaces $[17]$, might play a major causal role in cerebral vasospasm. However, other factors are also likely to be implicated in the complex pathophysiology of cerebral vasoconstriction and subsequent ischemia following SAH [22]. Leukotrienes are synthesized from arachidonic acid mainly through a 5lipoxygenase enzyme which is contained in neutrophils. Neutrophils are capable of synthesizing leukotrienes as well as taking up platelet-derived leukotrienes $[9,10]$.

Previous studies have shown a good correlation between the number of leukocytes and the extent of tissue damage following myocardial $[5,7,8,15,16]$ and cerebral [13] ischemia. Accordingly, total WBC counts were observed to be a good prognostic indicator in acute stroke patients [13]. 
In the present cases, admission WBC counts appeared to be mildly elevated. This is a relatively common finding early after the occurrence of SAH [12], which probably reflects a mild sympathetic overactivity following a $\mathrm{SAH}$ as a nonspecific stress reaction [1].

The WBC counts tended to be more elevated in patients graded worse, a fact already observed by other investigators $[11,12]$. Unlike other factors such as blood pressure level, number of previous hemorrhages, and angiographic vasospasm, the admission WBC counts failed to show a prognostic value as to the development of ICs in the patients in this study.

As already observed by other investigators [11], the total WBC counts rose subsequently in patients deteriorating because of ICs. In the present study, the increase in the cell count was statistically significant. It must be stressed that steroids were administered to these patients following the initial biochemical and clinical evaluation as a part of the management protocol. Corticosteroids might increase the neutrophil count by preventing their egress from the circulation [11].

However, this significant increase in the WBC counts paralleled the clinical deterioration and was not observed in those patients who did not suffer from ICs. Furthermore, surgical timing was similar in the two groups of patients, a fact that seems to exclude surgical trauma as being a major factor in the observed elevation of the cell count values. Infectious complications were in fact overrepresented in patients with ICs, an expected fact which might have influenced the cell count values. However, the elevation in WBC count definitely preceded the clinical manifestations of the infectious complications.

In this study, the time-course of changes in the cell count of patients suffering from ICs closely resembles that of the increase in body temperature observed by Rousseaux et al [17] in their cases with cerebral vasospasm and delayed neurological dysfunction following $\mathrm{SAH}$. Therefore, it would appear that leukocytosis and fever occurring in patients with SAH and with symptoms of ischemia might share a common cause. Rousseaux et al contemplated a role for prostaglandins as a causal possibility, but prostaglandins do not seem to influence the WBC count. Other factors capable of causing both leukocytosis and fever should be considered, such as cerebral tissue wasting and/or sympathetic overactivity which would occur in SAH patients with ICs [11].

In conclusion, the present study shows that admission leukocytosis is not a prognostic indicator of the subsequent occurrence of ICs in patients with ruptured intracranial aneurysms. The increase in the total WBC counts observed at the time of neurological deterioration might indicate initial structural damage of the brain tissue and/or an increased sympathetic and adrenocortical activity [11], which might contribute to the pro- duction of cerebral vasospasm after SAH "per se" $[1,11,23]$. Whatever the case may be, the present data do not prove (but do not exclude) that neutrophils may have a direct causal role in the pathogenesis of ICs of $\mathrm{SAH}$, perhaps mediated through leukotrienes $\mathrm{C}_{4}$ and $\mathrm{D}_{4}$, which are known to increase vascular permeability [2]. Further studies are required, and should include serial determinations of leukotriene concentrations in the serum and/or cerebrospinal fluid of patients with SAH.

This paper has been presented in part at the XXXVII annual meeting of the Scandinavian Neurosurgical Society, Aalborg, Denmark, June 1985.

\section{References}

1. Benedict CR, Phil (Oxon) D, Loach MA. Sympathetic nervous system activity in patients with subarachnoid hemorrage. Stroke 1978;9:237-44.

2. Dahlen SE, Bjork J, Hedqvist P, Arfors KE, Hammarstrom S, Samuelsson B. Leukotrienes promote plasma leakage and leukocyte adhesion in postcapillary venules. In vivo effect with relevance to the acute inflammatory response. Proc Natl Acad Sci USA $1981 ; 78: 3887-91$.

3. Guidetti B. Results of microsurgical treatment of intracranial anterior circle saccular aneurysms. In: Barnett HJM, Paoletti P, Flam ES, Brambilla G. Cerebrovascular diseases: new trends in surgical and medical aspects. New York: Elsevier North-Holland Biomedical Press, 1981:297-311.

4. Guidetti B, Spallone A. The role of antifibrinolytic therapy in the preoperative management of recently ruptured intracranial aneurysms. Surg Neurol 1981;15:239-48.

5. Hughes WL, Kalbfleisch JM, Brandt EN, Costloe JP. Myocardial infarction prognoses by discriminant analysis. Arch Intern Med 1963;111:338-45.

6. Jarman DA, Du Boulay GH, Kendall B, Boillin DJ. Responses of baboon cerebral and extra cerebral arteries to prostacyclin and prostaglandin endoperoxide in vitro and in vivo. J Neurol Neurosurg Psychiatry 1979;42:677-86.

7. Jolly SR, Kane WT, Bailie MB. Reduction of reperfusion injury after canine myocardial ischemia by superoxide dismutase plus catalase (abstr). Circulation 1983;68 (Suppl 3):185.

8. Ksiezycka E, Hastie R, Maroko PR. Reduction in myocardial damage after experimental coronary artery occlusion by two techniques which deplete neutrophils (abstr). Circulation 1983;68 (Suppl 3):185.

9. Marcus AJ. Recent progress in the role of platelets in occlusive vascular disease. Stroke 1983;14:475-9.

10. Moufarrij NA, Little JR, Skrinska V, Lucas FU, Latchaw JP, Slugg RM, Lesser RP. Thromboxane synthetase inhibition in acute focal cerebral ischemia in cats. J Neurosurg 1984;61:1107-12.

11. Neil-Dwyer G, Cruickshank J. The blood leukocyre count and its prognostic significance in subarachnoid hemorrhage. Brain 1974;97:79-84.

12. Parkinson D, Stephensen $S$. Leukocytosis and subarachnoid hemorrhage. Surg Neurol 1984;21:132-4.

13. Pozzilli C, Lenzi GL, Argentino C, Bozzao L, Rasura M, Giubilei $\mathrm{F}$, Fieschi $\mathrm{C}$. Peripheral white blood cell count in cerebral ischemic infarction. Acta Neurol Scand 1985;71:396-400. 
14. Quintana L, Konda R, Ishibashi Y, Yoshimoto T, Suzuki J. The effect of prostacyclin on cerebral vasospasm: an experimental study. Acta Neurochir (Wien) 1982;62:187-93.

15. Rao PS, Cohen MV, Mueller HS. Transcardiac production of thromboxane: prostacyclin and free radicals in early experimental ischemia. Circulation 1983;68 (Suppl 3):185.

16. Romson J, Hook BG, Kunkel SL, Abrams GD, Schork MA, Lucchesi $B$. Reduction of the extent of ischemic myocardial injury by neutrophil depletion in dog. Circulation 1983;67:1016-23.

17. Rousseaux P, Scherpereel B, Bernard MH, Graftieaux JP, Guyot $\mathrm{JF}$. Fever and cerebral vasospasm in ruptured intracranial aneurysms. Surg Neurol 1980;14:459-65.

18. Sano K, Asano T,'Tanishima T, Sasaki T. Lipidic peroxidation as a cause of cerebral vasospasm. Neurol Res 1980;2:253-72.

19. Sasaki T, Wakai S, Asano T, Takakuna K, Sano K. Prevention of cerebral vasospasm after $\mathrm{SAH}$ with a thromboxane synthetase inhibitor, OKY-1581. J Neurosurg 1982;57:74-82.

20. Spallone A. Overall management results of recently ruptured intracranial aneurysms. Phronesis 1982;3:349-53.

21. Suzuki S, Sobata E, Iwabuchi T. Prevention of cerebral ischemic symptoms in cerebral vasospasm with tapidil, an antagonist, and selective synthesis inhibitor thromboxane $A_{2}$. Neurosurgery 1981;9:679-85.

22. Von Holst H, Granstrom E, Hammarstrom S, Samuelsson B, Steiner $\mathrm{L}$. Effect of leukotrienes $\mathrm{C}_{4}, \mathrm{D}_{4}$, prostacyclin and thromboxane $A_{2}$ on isolated human cerebral arteries. Acta Neurochir (Wien) 1982;62:177-85

23. Walter $P$, Neil-Dwyer $G$, Cruickshank JM. Beneficial effects of adrenergic blockade in patients with subarachnoid hemorrhage. Med J 1982;284:1661-4. 